\title{
Regulation of forkhead box 01 (FOXO1) by protein kinase $B$ and glucocorticoids: different mechanisms of induction of beta cell death in vitro
}

\author{
G. Kaiser • F. Gerst • D. Michael • S. Berchtold • \\ B. Friedrich • N. Strutz-Seebohm • F. Lang • \\ H.-U. Häring • S. Ullrich
}

Received: 21 September 2012 / Accepted: 29 January 2013 / Published online: 23 February 2013

(C) Springer-Verlag Berlin Heidelberg 2013

\begin{abstract}
Aims/hypothesis In steroid diabetes insulin secretion does not adequately compensate for enhanced hepatic gluconeogenesis and peripheral insulin resistance. Previous studies suggest that activation of the transcription factor forkhead box O1 (FOXO1) contributes to glucocorticoid-induced beta cell death. This study examines the role and regulation of FOXO1 in insulin-secreting cells.
\end{abstract}

Electronic supplementary material The online version of this article (doi:10.1007/s00125-013-2863-7) contains peer-reviewed but unedited supplementary material, which is available to authorised users.

G. Kaiser · D. Michael • B. Friedrich · H.-U. Häring •

S. Ullrich $(\bowtie)$

Division of Endocrinology, Diabetology, Vascular Medicine, Nephrology and Clinical Chemistry, Department of Internal

Medicine IV, University of Tübingen, Otfried-Müller-Strasse 10,

72076 Tübingen, Germany

e-mail: susanne.ullrich@med.uni-tuebingen.de

G. Kaiser $\cdot$ F. Gerst $\cdot$ H.-U. Häring $\cdot$ S. Ullrich

Institute for Diabetes Research and Metabolic Diseases

of the Helmholtz Centre Munich at the University of Tübingen,

Partner of the German Centre for Diabetes Research,

Tübingen, Germany

D. Michael $\cdot$ S. Berchtold $\cdot$ N. Strutz-Seebohm $\cdot$ F. Lang

Institute of Physiology, University of Tübingen,

Tübingen, Germany

Present Address:

S. Berchtold

Division of Gastroenterology, Department of Internal Medicine,

University of Tübingen, Tübingen, Germany

Present Address:

N. Strutz-Seebohm

Institute for Genetics of Heart Diseases (IfGH),

Department of Cardiovascular Medicine,

University Hospital Münster, Münster, Germany
Methods INS-1E cells and mouse islet cells were cultured in the presence of dexamethasone. Signalling pathways were modified pharmacologically or by small interfering (si) RNA-mediated inhibition of protein synthesis. Changes in protein abundance and phosphorylation were analysed by western blotting, and subcellular localisation was assessed using confocal microscopy. Transcript levels were examined by RT-PCR.

Results Surprisingly, downregulation of FOXO1 by siRNA did not affect dexamethasone-induced apoptosis or Bim expression, but it prevented the effects of the pan protein kinase B (AKT) inhibitor (Akti-1/2). Indeed, dexamethasone and Akti-1/2 synergistically increased beta cell death and Bim expression. Akti-1/2 triggered dephosphorylation and nuclear translocation of FOXO1. Glucocorticoidreceptor activation stimulated Foxol transcription, but FOXO1 phosphorylation was unchanged and the cytosolic concentration of FOXO1 remained high in relation to its nuclear concentration. However, subcellular fractionation revealed a significant increase in both cytosolic and nuclear FOXO1 compared with untreated cells. Dexamethasone diminished $P d x 1$ mRNA level, an effect which was not reversed by siRNA against Foxol. Downregulation of AKT isoforms and serum/glucocorticoid-regulated kinase 1 (SGK1) suggests that only sustained suppression of all three AKT isoforms caused dephosphorylation and nuclear accumulation of FOXO1.

Conclusions/interpretation This study reveals that FOXO1 is not the main mediator of glucocorticoidreceptor-induced beta cell apoptosis, but rather that it escalates beta cell death when AKT activity is inhibited by distinct pathways.

Keywords AKT isoforms - Apoptosis - Dexamethasone FOXO1 · Glucocorticoids · Insulin-secreting cells · SGK1 


$\begin{array}{ll}\text { Abbreviations } \\ \text { AKT } & \text { Protein kinase B } \\ \text { Akti-1/2 } & \text { AKT inhibitor-1/2 } \\ \text { BAD } & \text { BCL2-associated agonist of cell death } \\ \text { FOXO1 } & \text { Forkhead box O1 } \\ \text { p- } & \text { Phosphorylated } \\ \text { PARP } & \text { Poly(ADP-ribose) polymerase family, member 1 } \\ \text { SGKi } & \text { SGK1 inhibitor GSK650394 } \\ \text { SGK1 } & \text { Serum/glucocorticoid-regulated kinase 1 } \\ \text { si } & \text { Small interfering }\end{array}$

\section{Introduction}

In clinical therapy, glucocorticoids such as the synthetic agent dexamethasone are commonly used in autoimmune and anti-inflammatory disease management. A frequent side effect of long-term and high-dose glucocorticoid treatment is the development of steroid diabetes [1]. In addition to an increase in hepatic glucose production [2] and peripheral insulin resistance [3], glucocorticoids impair beta cell function [4]. The underlying mechanism of glucocorticoidinduced beta cell dysfunction is not well understood. Many observations point to a crucial role of the transcription factor forkhead box O1 (FOXO1) in beta cell failure [5]. However, the role of FOXO1 in the maintenance of beta cell function and appropriate beta cell mass is controversial [6]. On one hand, FOXO1-mediated inhibition of the beta cell-specific transcription factor $P d x 1$ impairs beta cell neogenesis and contributes to a reduction of beta cell mass [7]. On the other hand, FOXO1 induces NeuroD and MafA expression, which encode two transcription factors essential for insulin gene transcription [8]. A reduction in FOXO1 protein protects against beta cell death induced by the synthetic glucocorticoid dexamethasone, suggesting that FOXO1 activation might mediate the pro-apoptotic effects of glucocorticoids [9]. In insulin-secreting cells FOXO1 stays under the control of insulin/IGF-1 signalling through IRS-2 and protein kinase B (AKT) [10]. Previously, we described that dexamethasone reduces phosphorylation of AKT and of the proapoptotic protein BCL2-associated agonist of cell death (BAD), triggering the intrinsic apoptotic pathway [11].

Further effects of glucocorticoids on beta cell function comprise inhibition of insulin biosynthesis and insulin secretion [11-16]. Inhibition of insulin secretion is at least partly mediated by increased potassium channel activity [12]. The mechanism involves $\alpha_{2}$-adrenoceptor activation and serum/glucocorticoid-regulated kinase 1 (SGK1) induction [17]. SGK1, a serum- and glucocorticoid-inducible kinase with $75 \%$ structural homology with AKT, increases the activity of voltage-dependent potassium channels $[18,19]$. Interestingly, a genetic polymorphism downstream of SGK1 3'untranslated region (UTR) associates with increased insulin secretion and reduced risk of developing diabetes mellitus, which is indicative of a regulatory role of SGK1 in human beta cell function [20]. SGK1 and the other two isoforms, SGK2 and SGK3, are similar to AKT in that they are stimulated by insulin/IGF-1 receptor signalling and share substrates. A common substrate of SGK and AKT isoforms is the transcription factor FOXO1. AKT phosphorylates FOXO1 at Thr24, Ser256 and Ser319, while SGK1 preferentially phosphorylates its Ser256 and Ser319 residues [21-23]. Phosphorylation of FOXO1 is believed to be one of the main antiapoptotic signals downstream of AKT [24]. Phosphorylated FOXO1 is confined into the cytosol, while inhibition of AKT allows the translocation of the transcription factor into nuclei and activation of FOXO1-dependent pro-apoptotic genes.

This study questions the role of AKT isoforms and SGK1 on FOXO1 regulation and its impact on glucocorticoidinduced beta cell death.

\section{Methods}

Reagents Antibodies against FOXO1 (cat no. 2880), phosphorylated (p)-FOXO1 (Ser256, cat no. 9461), AKT (cat no. 9272), p-AKT (Ser473, cat no. 9271), AKT1 (cat no. 2967), poly(ADP-ribose) polymerase family, member 1 (PARP) (cat no. 9542), SGK1 (cat no. 3272) and $\alpha / \beta$-tubulin (cat no. 2148) were from Cell Signaling Technology (Beverly, MA, USA). Antibodies against p-FOXO1 (Ser319, cat no. sc-101682), glyceraldehyde 3-phosphate dehydrogenase (GAPDH)-horseradish peroxidase (HRP) (cat no. 20357-HRP) and Histone H1 (cat no. sc-10806) were purchased from Santa Cruz Biotechnology (Santa Cruz, CA, USA). Peroxidase-linked donkey anti-rabbit IgG was from GE Healthcare (GE Healthcare Europe, Freiburg, Germany). Alexa Fluor 488 anti-rabbit IgG was obtained from Invitrogen (Karlsruhe, Germany). The antibody for SGK1 used for immunohistochemical staining was made by Pineda (Berlin, Germany).

Cell culture media were obtained from Gibco (Invitrogen) and FCS from Biochrom (Berlin, Germany). The protein assay was purchased from BioRad Laboratories (München, Germany), the protein ladder from Invitrogen. AKT inhibitor1/2 (Akti-1/2) was purchased from Merck Chemicals (Nottingham, UK), GSK650394 from Tocris Bioscience (R\&D Systems Wiesbaden-Nordenstadt, Germany) and nuclear dye TOPRO3 from Invitrogen. All other chemicals were from Sigma-Aldrich (Deisenhofen, Germany) and of analytical grade unless otherwise stated.

INS-1E cell culture and transfection Cells of the rat insulinoma cell line INS-1E (kindly provided by C. B. Wollheim, University of Geneva, Switzerland) were cultured in HEPES-buffered RPMI 1640 supplemented with 10\% (vol./vol.) FCS, 10 mmol/l HEPES, 2 mmol/l L-glutamine, 
$1 \mathrm{mmol} / 1$ sodium pyruvate and $10 \mu \mathrm{mol} / 1 \beta$-mercaptoethanol, as described previously [18]. The culture medium contained $11 \mathrm{mmol} / \mathrm{l}$ glucose.

Cells were transfected with the plasmids $\mathrm{h} S \mathrm{~g} k 1^{\mathrm{SD}} / \mathrm{pIRES} 2-$ EGFP and $h S g k 1^{\mathrm{KN}} / \mathrm{pIRES} 2-\mathrm{EGFP}\left(\mathrm{Sgk} 1^{\mathrm{SD}}\right.$ encodes the constitutively active form and $S g k 1^{\mathrm{KN}}$ encodes the kinase dead form of SGK1, respectively [Clontech, Saint-Germain-enLaye, France]) or small interfering (si)RNA directed against Sgk1 (Qiagen, Hilden, Germany), Akt1, Akt2, Akt3, Foxo1 (siGenome smart pool) or siGLO RISC-Free Control siRNA using DharmaFect Transfection Reagent 3 (Dharmacon, Chicago, USA). Cells were analysed 1 day after transfection with plasmid and 2 days after transfection with siRNA.

Mouse islet preparations Mouse islets from adult C57BL/6 mice were isolated using collagenase digestion $(1 \mathrm{mg} / \mathrm{ml}$ collagenase, Serva, Heidelberg, Germany). Single islet cells were prepared by digestion with trypsin-EDTA in PBS for 4-8 $\mathrm{min}$ at $37^{\circ} \mathrm{C}$ and thereafter seeded on polyornithinecoated $(0.001 \%$ (wt/vol.)) glass cover slips. Islets and islet cells were cultured overnight in the same medium as INS-1E cells without addition of $\beta$-mercaptoethanol. After medium change, cells and islets were cultured in the presence of dexamethasone, $100 \mathrm{nmol} / \mathrm{l}$ for $24 \mathrm{~h}$. Akti-1/2, $3 \mu \mathrm{mol} / \mathrm{l}$, was added for the last $30 \mathrm{~min}$.

Immunohistochemical staining and TUNEL assay INS-1E cells or mouse islet cells were cultured in the presence of test substances, as indicated. Cells were fixed with $4 \%$ paraformaldehyde in PBS for $1 \mathrm{~h}$, permeabilised with $0.2 \%$ (vol./vol.) Triton X-100 for 2 min on ice, blocked with $10 \%$ (vol./vol.) FCS for $45 \mathrm{~min}$ at room temperature and incubated overnight with a primary antibody against FOXO1 (1:100) or SGK1 (1:100). After incubation with a secondary antibody (1:400 Alexa Fluor 488 anti-rabbit IgG) for $1 \mathrm{~h}$, nuclei were stained with $1 \mu \mathrm{mol} / \mathrm{l}$ TO-PRO3 in PBS for $1 \mathrm{~h}$. TUNEL staining was performed according to the instructions of the manufacturer (Roche Diagnostics, Mannheim, Germany). Thereafter, nuclei were stained with DAPI, $0.1 \mu \mathrm{g} / \mathrm{ml}$ in PBS, for $30 \mathrm{~min}$ at room temperature. Fluorescence was visualised using a laser scanning microscope (Leica Microsystems, Wetzlar, Germany).

Western blotting Cells were seeded and treated with test substances as indicated for each experiment. For experiments with the AKT inhibitor Akti-1/2 and the SGK1 inhibitor GSK650394 (SGKi), cells were pre-incubated for $1 \mathrm{~h}$ in KRB supplemented with $2.8 \mathrm{mmol} / 1$ glucose and $0.1 \%$ (wt/vol.) BSA (fraction V); dexamethasone was present throughout the preincubation. For western blotting, cells were lysed in buffer containing $125 \mathrm{mmol} / \mathrm{l} \mathrm{NaCl}, 1 \%$ (vol./vol.) Triton X-100, 0.5\% (wt/vol.) Na-deoxycholate, $0.1 \%$ (wt/vol.) SDS, 10 mmol/1 EDTA, 25 mmol/1 HEPES pH 7.3, 10 mmol/1
NaPP, $10 \mathrm{mmol} / 1 \mathrm{NaF}, 1 \mathrm{mmol} / \mathrm{l} \mathrm{Na}$-vanadate, $10 \mu \mathrm{g} / \mathrm{ml}$ pepstatin A, $10 \mu \mathrm{g} / \mathrm{ml}$ aprotinin and $0.1 \mathrm{mmol} / 1$ phenylmethylsulfonylfluoride (PMSF) as described previously [11]. Proteins $(30 \mu \mathrm{g})$ of a $10,000 \mathrm{~g}$ supernatant fraction were subjected to SDS-PAGE and blotted on nitrocellulose membranes (Schleicher and Schuell, Dassel, Germany). Antibodies were diluted 1:1000 in Tris-buffered saline (TBS) supplemented with $0.15 \%$ (vol./vol.) Tween and 5\% (wt/vol.) BSA or 5\% (wt/vol.) milk. Quantification of western blots was performed by measuring the optical densities of the respective bands on the film using ImageJ (NIH, Bethesda, MD, USA). For nuclear and cytosolic cell fractionation a commercial kit (Thermo Scientific; Rockford, IL, USA) was used and $30 \mu \mathrm{g}$ protein of the cytosolic fraction and $15 \mu \mathrm{g}$ protein of the nuclear fraction were loaded onto western blots.

Quantitative real-time RT-PCR analysis INS-1E cells were cultured under standard conditions and treated with substances as indicated. Thereafter, cells were lysed and total RNA isolated using a commercial kit (Qiagen). After cDNA synthesis using Oligo(dT)12-18 as primer (Roche Diagnostics), specific PCR was performed with the Light Cycler Fast Start Master SybrRGreen method (Roche Diagnostics) and the Light Cycler Primer Set for Murine/Rat GAPDH (Roche Diagnostics) as external standard. The primers are listed in electronic supplementary material (ESM) Table 1.

Quantification of mRNA levels of mouse islets was performed with the Light Cycler System LC480 and the LC480 Probes Master (Roche Diagnostics). Gene expression relative to the housekeeping gene Rps 13 was quantified by the $2^{-\Delta \Delta C_{t}}$ method. The respective primers are listed in ESM Table 2.

Statistics Data are provided as mean \pm SEM; $n$ represents the number of observations of at least three independent experiments. All data were tested for significance using Student's $t$ test, ANOVA and Newman-Keuls test as post hoc test where appropriate. Results with $p<0.05$ were considered statistically significant.

\section{Results}

Activation of FOXO1 is not required for dexamethasoneinduced cell death To examine whether FOXO1 contributes to dexamethasone-induced cell death, FOXO1 synthesis was downregulated by up to $86 \%$ in INS-1E cells using the siRNA strategy (Fig. 1a, b). Treatment of cells with the pan AKT inhibitor Akti-1/2 was used as a positive control for AKT-dependent FOXO1 regulation [25]. Apoptosis was examined by two independent methods, by immunohistochemical staining of TUNEL-positive cells and by detection of cleaved PARP, which reflects the activation of 


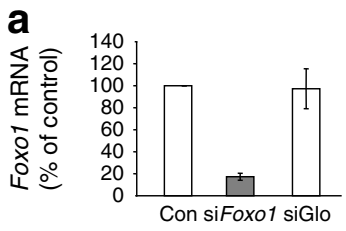

b
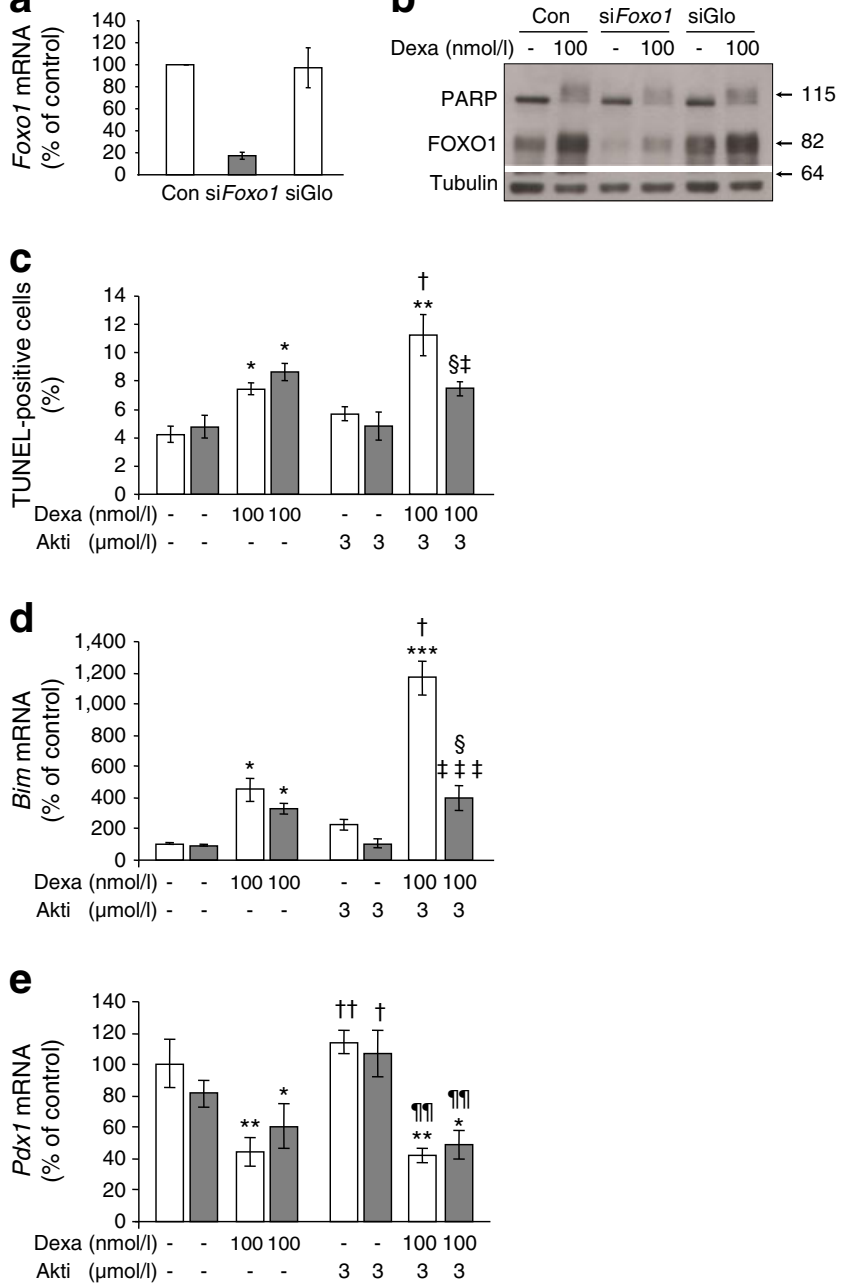

Fig. 1 Activation of FOXO1 is not required for dexamethasone-induced cell death. INS-1E cells were transfected with siRNA against Foxol (grey bars) or siGLO (white bars) and treated with dexamethasone, $100 \mathrm{nmol} / 1$ for 1 day, and with Akti-1/2, $3 \mu \mathrm{mol} / 1$, in the presence of dexamethasone for $2 \mathrm{~h}$ for TUNEL and for $8 \mathrm{~h}$ for mRNA analysis, as indicated and described in the Methods. (a) Relative amounts of Foxol mRNA are expressed as mean \pm SEM of $n=5$ independent experiments. (b) A representative western blot for PARP and FOXO1. Tubulin was used as loading control. (c) The percentage of TUNEL-positive cells is expressed as mean \pm SEM of $n=5$ independent experiments. (d) Bim and (e) $P d x 1$ mRNA levels are expressed as mean \pm SEM of $n=4$ independent experiments. The content of mRNA under control was set to $100 \%{ }^{*} p<0.05$ and $* * p<0.01$ denote significance compared with siGlo untreated (first bar of each graph); ${ }^{\dagger} p<0.05$ and ${ }^{\dagger \dagger} p<0.01$ denote significance compared with siGlo dexamethasone-treated; ${ }^{\ddagger} p<0.05$ and ${ }^{+\$ t} p<0.001$ denote significance compared with siGlo treated with dexamethasone and Akti-1/2; $\S_{p}<0.05$ denotes significance compared with siRNA Foxol treated with Akti-1/2; ${ }^{\uparrow \uparrow} p<0.01$ denotes significance compared with the respective Akti-1/2 treated cells; Akti, Akti-1/2; Con, control; Dexa, dexamethasone; siFoxo1, cells transfected with siRNA against Foxol

caspase-3 [26]. In FOXO1-deficient cells Foxo3 and Foxo4 mRNA remained unchanged (data not shown). The successful inhibition of FOXO1 synthesis did not prevent either the dexamethasone-induced degradation of PARP or the increase in the number of TUNEL-positive nuclei (Fig. 1b, c). The significant augmentation of dexamethasone-induced apoptosis by the AKT inhibitor Akti-1/2 ( $3 \mu \mathrm{mol} / \mathrm{l}$ for $2 \mathrm{~h})$, however, was completely abrogated by siRNA against Foxol (Fig. 1c). In accordance, dexamethasone increased Bim mRNA levels in a FOXO1-independent manner, while the effect of Akti- $1 / 2$ on Bim mRNA was abrogated in FOXO1-deficient cells (Fig. 1d). Furthermore, dexamethasone reduced $P d x 1$ mRNA levels independently of FOXO1, while Akti-1/2 did not alter mRNA levels of $P d x 1$ (Fig. 1e). These results suggest that glucocorticoid-receptor activation triggers apoptotic beta cell death in a FOXO1-independent manner, which may further sensitise the cells toward FOXO1-mediated apoptotic signals.

Akti-1/2 but not dexamethasone inhibited phosphorylation and promoted nuclear accumulation of FOXO1 As AKT activity determines phosphorylation and subcellular localisation of FOXO1 and previous observations show that dexamethasone inhibits phosphorylation of AKT, phosphorylation of FOXO1 and its subcellular distribution were examined in dexamethasone-treated INS-1E cells. Surprisingly, phosphorylation of FOXO1 at Ser256 and Ser319 was significantly higher in dexamethasone-treated $(100 \mathrm{nmol} / \mathrm{l}$ for $24 \mathrm{~h}) \mathrm{com}-$ pared with control whole-INS-1E-cell lysates (Fig. 2a, b). The increase in FOXO1 protein occurred in parallel with its phosphorylation. Consequently, dexamethasone did not change the degree of phosphorylation of FOXO1 (Fig. 2c-e). As against this, Akti-1/2 ( $3 \mu \mathrm{mol} / 1$ for $30 \mathrm{~min})$ strongly inhibited phosphorylation of FOXO1 (Fig. 2f). In control as well as in dexamethasone-treated cells (100 nmol/1 for 4, 24 and $48 \mathrm{~h}$ ) the concentration of FOXO1 was higher in the cytosolic compartments than in the nuclei, as confirmed by confocal microscopy (Fig. 3a). In contrast, exposure to Akti-1/2 for as short a period as $30 \mathrm{~min}$ induced nuclear accumulation of FOXO1 (Fig. 3b). These observations confirm that Akti-1/2 activates FOXO1 while dexamethasone does not.

Intriguingly, subcellular fractionation suggests that the amount of FOXO1 increased in the cytosolic fraction and also in the nuclear fraction on dexamethasone treatment (ESM Fig. 1a). Treatment with Akti-1/2 ( $3 \mu \mathrm{mol} / 1$ for $30 \mathrm{~min}$ ) resulted in translocation of FOXO1 as the cytosolic amount of FOXO1 decreased while the nuclear amount increased. Additionally, in dexamethasone-exposed INS1E cells AKT phosphorylation was significantly reduced, in line with previous observations (ESM Fig. 1b).

To confirm that regulation of FOXO1 by dexamethasone and Akti-1/2 in INS-1E cells translates to primary islet cells, comparable experiments were performed with islets isolated from adult mice (Fig. 4). In mouse islet cells cultured in the presence of dexamethasone (100 nmol/l for $24 \mathrm{~h})$, FOXO1 immunoreactivity was more prominent in the cytosol than in nuclei, while after exposure to Akti-1/2 ( $3 \mu \mathrm{mol} / 1$ for $30 \mathrm{~min})$ FOXO1 accumulated in nuclei (Fig. 4a). Dexamethasone treatment of mouse islets (100 nmol/l for $24 \mathrm{~h})$ significantly 
a

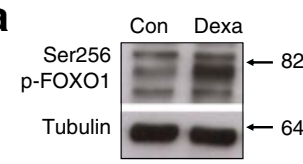

b

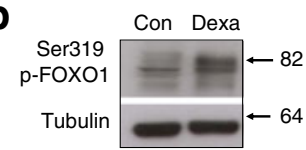

C

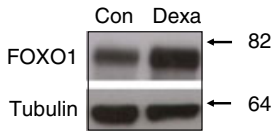

d

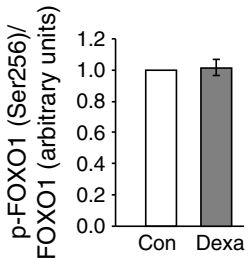

f

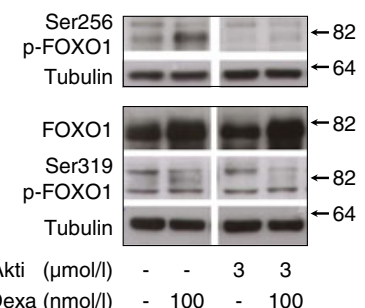

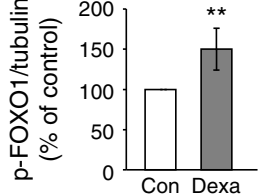
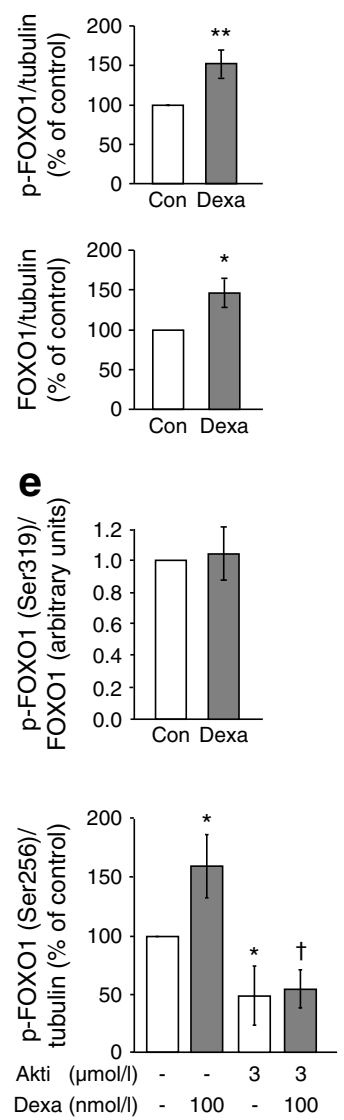

Fig. 2 Akti-1/2 but not dexamethasone inhibited phosphorylation of FOXO1. Cells were treated with dexamethasone, $100 \mathrm{nmol} / 1$ for $24 \mathrm{~h}$, and Akti-1/2, $3 \mu \mathrm{mol} / 1$ for $30 \mathrm{~min}$, as indicated and described in the Methods. (a-c, f) Representative western blots and mean \pm SEM of three to five independent experiments for p-FOXO1 (Ser256) (a), p-FOXO1 (Ser319) (b), FOXO1 (c) and p-FOXO1 (Ser256), FOXO1 and p-FOXO1 (Ser319) (f). Tubulin was used as loading control. The ratio of p-FOXO1 (Ser256) (d) and p-FOXO1 (Ser319) (e) to FOXO1 protein is presented as mean \pm SEM of $n=6$ independent experiments. ${ }^{*} p<0.05$ and ${ }^{*} p<0.01$ denote significance compared with control; ${ }^{\dagger} p<0.05$ denotes significance compared with dexamethasone. Akti, Akti-1/2; Con, control; Dexa, dexamethasone

increased Foxo1 (Fig. 4b), Foxo3 (Fig. 4c) and Bim (Fig. 4d) mRNA levels but reduced $P d x 1$ (Fig. 4e) mRNA levels as in INS-1E cells. The mRNA concentration of Foxol was notably higher (200-fold) than that of Foxo3. Thus, dexamethasone exerts similar effects in INS-1E cells and mouse islets.

Specific effects of glucocorticoid-receptor activation on Foxo, Akt and Sgk isoforms To examine the mechanism underlying increased FOXO1 protein in cells exposed to an excess of glucocorticoids, the effect of dexamethasone
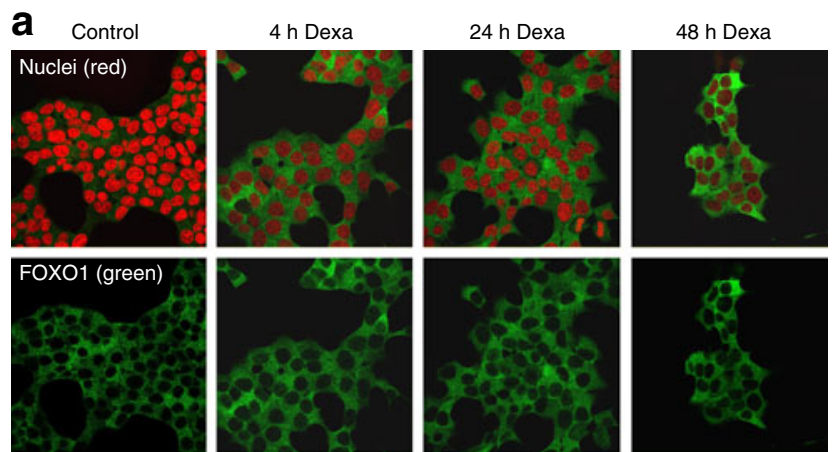

b
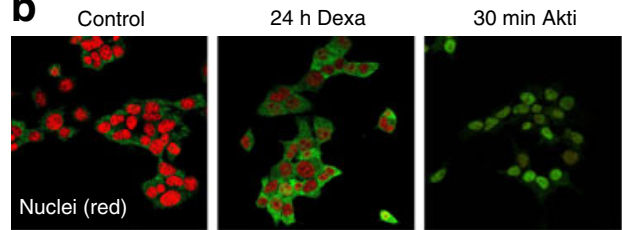

24 h Dexa+30 min Akti
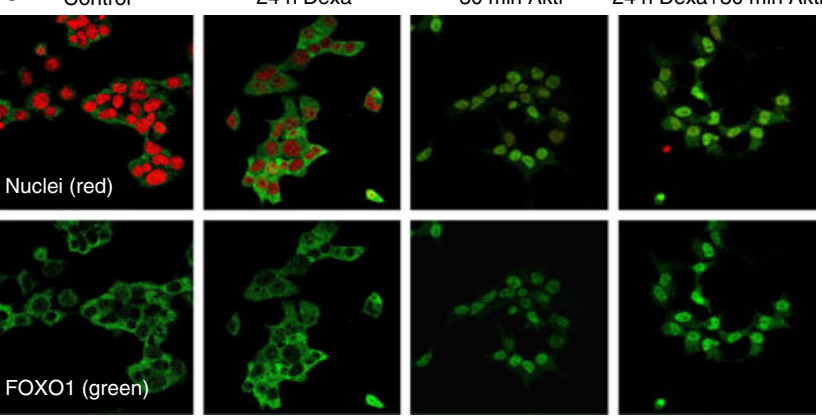

Fig. 3 The inhibitor Akti-1/2, but not dexamethasone, promotes nuclear accumulation of FOXO1. (a) INS-1E cells were cultured under standard conditions and treated with dexamethasone for the indicated time. (b) Cells were treated as described in Fig. 2. Representative pictures of immunostaining of FOXO1 (green) using confocal microscopy are shown. Nuclei are stained in red. Akti, Akti-1/2; Dexa, dexamethasone

on Foxo1 transcript levels was evaluated by semiquantitative analysis of mRNA levels in the absence and presence of the glucocorticoid-receptor inhibitor RU486 (ESM Figs 2, 3). In INS-1E cells, Foxo1 mRNA was as abundant as Foxo 3 mRNA, whereas the level of Foxo 4 mRNA was 30fold lower (ESM Fig. 2a). Glucocorticoid treatment specifically increased Foxol mRNA within 4 h (ESM Fig. 2b). A 2.5 -fold increase observed throughout the treatment (24 and $48 \mathrm{~h}$ ) was abrogated on exposure to the glucocorticoidreceptor antagonist RU486 (mifepristone, $10 \mu \mathrm{mol} / 1$ [ESM Fig. 3a]). Foxo3 and Foxo 4 mRNA levels increased significantly only after prolonged exposure $(24-48 \mathrm{~h})$ to the glucocorticoid analogue (ESM Fig. 2c, d). These effects were again sensitive to mifepristone (ESM Fig. 3b, c).

In the next series of experiments mRNA levels of $A k t$ and $S g k$ isoforms were analysed to identify the enzymes responsible for phosphorylation of FOXO1 (ESM Fig. 2e-1). Control INS-1E cells expressed comparable levels of $A k t 1$ and $S g k 1$ mRNA (ESM Fig. 2e, i). Akt2 and Akt3 mRNA levels were tenfold and fivefold lower, respectively, than Akt1 mRNA levels (ESM Fig. 2e); $S g k 2$ and $S g k 3$ mRNA levels were 100-fold and tenfold lower, respectively, than $S g k 1$ mRNA levels (ESM Fig. 2i). Dexamethasone treatment resulted in a unique 20-fold increase in $S g k 1$ mRNA (ESM Fig. 2j). Accordingly, SGK1 protein was detected by 
Fig. 4 Effects of dexamethasone on Foxol, Foxo3, Bim and $P d x 1$ expression in mouse islets. Mouse islet cells were isolated and cultured as described in Methods. (a) Representative pictures of immunostaining of FOXO1 (green) and nuclei (red) using confocal microscopy; scale bar $50 \mu \mathrm{m}$. (b-e) Relative gene expressions of Foxol (b), Foxo 3 (c), $\operatorname{Bim}(\mathbf{d})$ and $P d x l$ (e) were determined by quantitative RTPCR and are expressed as mean \pm SEM of $n=4$ independent experiments. Rps 13 was used as housekeeping gene. For Bim and $P d x l$, mRNA content of untreated cells was set to $100 \%$. $* p<0.05$ and $* * p<0.01$ denote significance to untreated cells. Akti, Akti-1/2; Dexa, dexamethasone a
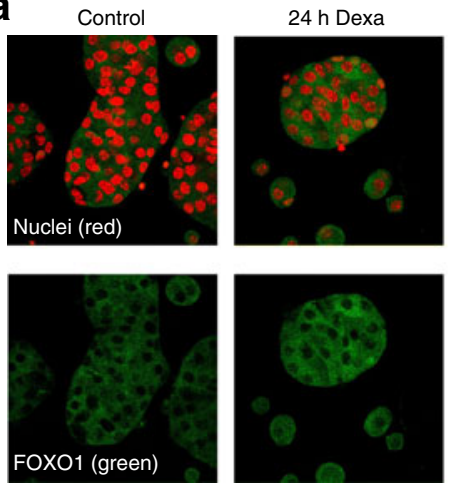

b

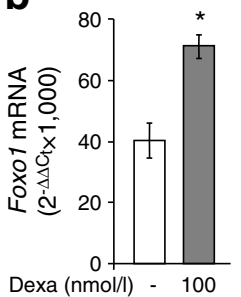

C

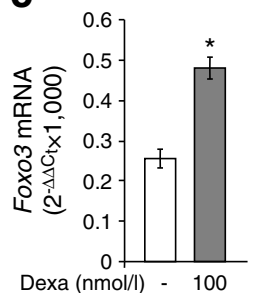

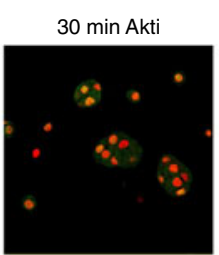
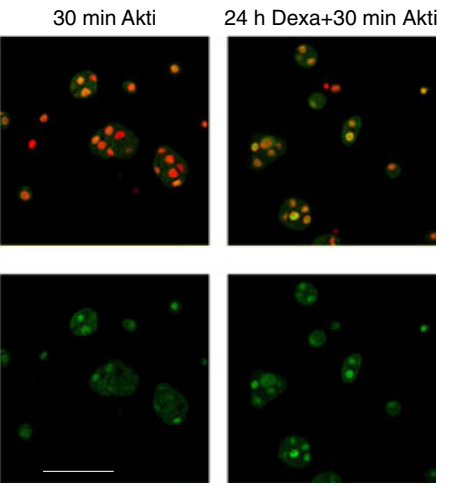

d

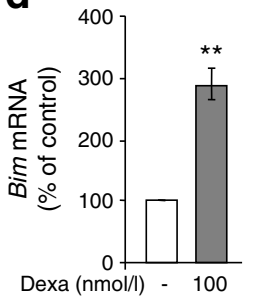

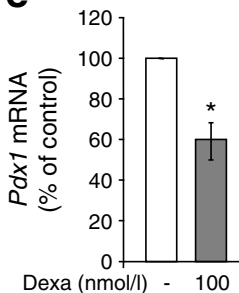

immunostaining in dexamethasone-treated, but not in control, INS-1E cells (ESM Fig. 3d). The relative mRNA concentrations of $S g k 2$ and $S g k 3$ (ESM Fig. 2k, 1) as well as of all three $A k t$ isoforms (ESM Fig. $2 \mathrm{f}-\mathrm{h}$ ) were not significantly altered by dexamethasone.

These findings suggest that increased FOXO1 protein is a result of specific glucocorticoid-receptor-mediated activation of transcription. FOXO1 phosphorylation and its cytosolic retention could be regulated by the action of multiple AKT and SGK isoforms, of which the most abundant are SGK1 and AKT1.

Phosphorylation of FOXO1 depended on AKT1, AKT2 and $A K T 3$ To examine whether the discrepancy between dexamethasone-induced inhibition of AKT phosphorylation and maintenance of FOXO1 phosphorylation could be explained by effects of distinct AKT isoforms, isoformspecific siRNAs were used to inhibit selectively Akt1, Akt2 and Akt3. Inhibition of the synthesis of individual AKT isoforms did not alter phosphorylation of FOXO1 (ESM Fig. 4a). Phosphorylation of FOXO1 persisted even when the synthesis of two AKT isoforms (AKT1 and AKT2 or AKT1 and AKT3 or AKT2 and AKT3) was simultaneously inhibited (ESM Fig. 4b). The individual mRNA levels were reduced to $16.0 \pm 1.1 \%$ for $A k t 1,21.1 \pm 2.2 \%$ for $A k t 2$ and $18.4 \pm 1.5 \%$ for Akt3 (ESM Table 3). Immunostaining of FOXO1 remained prominent in the cytosolic compartment, confirming phosphorylation of FOXO1 (Fig. 5a). Finally, the treatment of INS-1E cells with siRNA against all three $A k t$ isoforms, which resulted in an 80-90\% reduction of their transcript levels, significantly inhibited phosphorylation of FOXO1 (Fig. 5b) and increased its nuclear staining in control INS-1E cells (Fig. 5e). The dexamethasone-mediated increase in FOXO1 level was not affected by siRNAs against all $A k t$ isoforms (Fig. 5c), whereas phosphorylation of FOXO1 was significantly reduced but not abrogated (Fig. 5d). In conclusion, all three isoforms, AKT1, AKT2 and AKT3, phosphorylate and facilitate nuclear extrusion of FOXO1 and, via this, mediate the inhibition of its transcriptional activity in insulin-secreting cells.

FOXO1 phosphorylation was not sensitive to SGK1 abundance or activity Dexamethasone treatment induces $S g k 1$ expression and this kinase could contribute to FOXO1 inhibition in cells exposed to glucocorticoid excess. The role of SGK1 in the regulation of FOXO1 was clarified by specifically changing the activity and production of SGK1. Neither the inhibition of SGK1 activity by SGKi, $10 \mu \mathrm{mol} / 1$, nor the prevention of dexamethasone-induced upregulation of SGK1 with siRNA reduced phosphorylation of FOXO1 at Ser256 and Ser319 (ESM Fig. 5a, b). In accordance, neither the overexpression of kinase dead $S g k 1$ ( $S g k 1 \mathrm{KN})$ nor the overexpression of constitutively active $S g k 1$ ( $S g k 1 \mathrm{SD})$ affected phosphorylation of FOXO1 at Ser256 (ESM Fig. 5c). The analysis of the cellular distribution of FOXO1 by confocal microscopy confirmed that in cells treated with dexamethasone and SGKi, FOXO1 remained largely cytosolic (ESM Fig. 5d). These observations suggest that SGK1 does not account for cytosolic retention of FOXO1 in insulin-secreting cells under conditions of glucocorticoid excess.

In summary, beta cell death induced by glucocorticoids occurs in the absence of substantial activation of FOXO1, but is amplified when the overall activity of AKT is sufficiently reduced, resulting in the nuclear translocation and activation of FOXO1. 
a
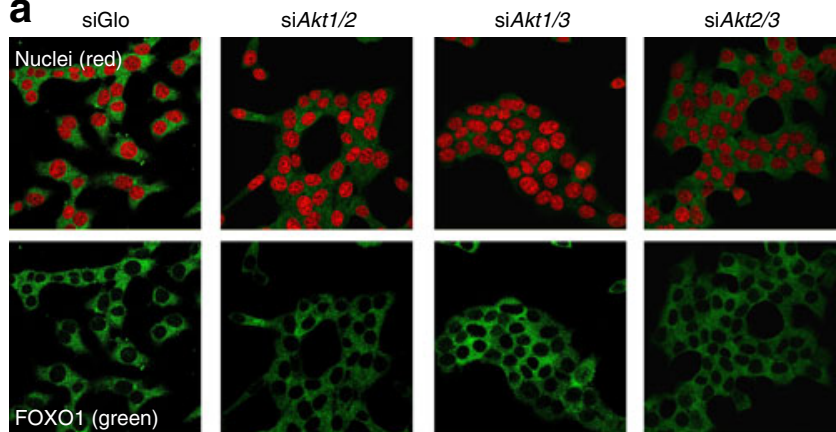

b
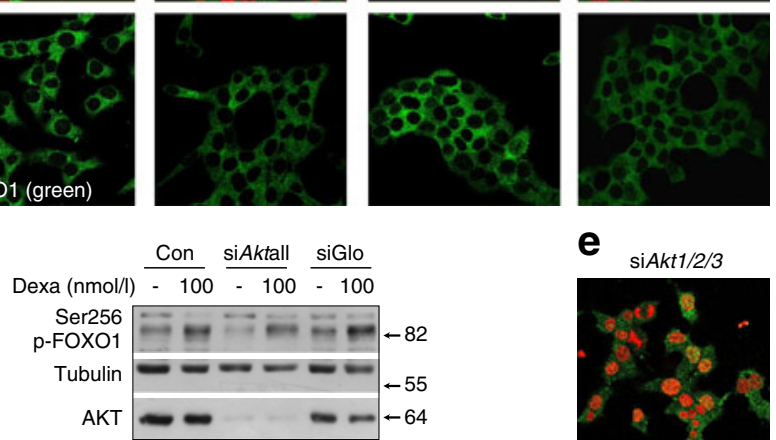

C

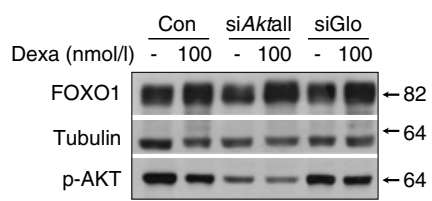

d

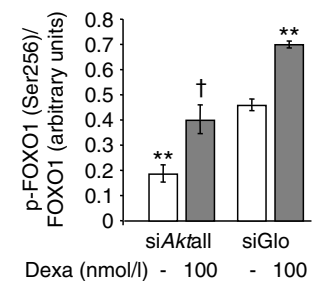

Fig. 5 Phosphorylation and nuclear accumulation of FOXO1 is dependent on AKT1, AKT2 and AKT3. (a-e) INS-1E cells were treated with siRNA as indicated and cultured under standard culture conditions $(\mathbf{a}, \mathbf{e})$ and in the presence of dexamethasone, $100 \mathrm{nmol} / 1$ for 1 day. (b-d) Representative pictures of immunostaining of FOXO1 (green) using confocal microscopy. Nuclei are stained in red. (a, e) Representative western blots of three independent experiments for p-FOXO1 (Ser256) (b) and FOXO1 (c) protein are shown. Tubulin was used as loading control. (d) The ratio of p-FOXO1 (Ser256) to FOXO1 protein is presented as mean \pm SEM of $n=3$ independent experiments. ${ }^{* *} p<0.01$ denotes significance compared with siGlo under control conditions; ${ }^{\dagger} p<0.05$ denotes significance compared with siGlo treated with dexamethasone. Dexa, dexamethasone; siAktall, cells transfected with siRNA against $A k t 1 / A k t 2 / A k t 3$ isoforms

\section{Discussion}

This study shows that glucocorticoids induce beta cell death independently of FOXO1, as downregulation of FOXO1 using the siRNA strategy did not reduce the rate of cell death induced by dexamethasone. The detailed analysis of posttranslational phosphorylation and subcellular distribution of FOXO1 suggests that FOXO1 remained phosphorylated and was largely localised in the cytosol even when apoptosis was significantly increased i.e. after $24 \mathrm{~h}$ exposure of INS-1E cells to dexamethasone. This observation is even more surprising as dexamethasone increased the expression of Foxol, confirming a previous study with INS- 1 cells showing that dexamethasone increases, while prolactin decreases, the transcript levels of Foxol [27]. Increased protein levels in combination with reduced phosphorylation and activation of FOXO1 after dexamethasone exposure has been described in a variety of cells, including hepatocytes, cardiomyocytes and tenocytes [28-30]. Interestingly, in tenocytes, dexamethasone-mediated inhibition of FOXO1 phosphorylation was counteracted by insulin. Previously, we described that IGF-1 reduces dexamethasone-induced cell death [16]. Thus, it is reasonable to speculate that insulin, which is secreted by INS-1E cells into the culture medium, may be responsible for sufficient activation of AKT and the subsequent phosphorylation and cytosolic localisation of FOXO1. Subcellular fractionation confirmed that the amount of FOXO1 increased in the cytosol after dexamethasone treatment. Intriguingly, the amount of FOXO1 in the nucleus was also increased when compared with untreated cells. There is a possibility that nuclear FOXO1 is not pro-apoptotic, as our results suggest that induction of apoptosis by dexamethasone occurs independently of FOXO1 [6].

Dexamethasone-induced cell death was significant even in the presence of secreted insulin, indicating that pathways other than FOXO1 signalling trigger apoptosis. Indeed, increased Bim expression induced by dexamethasone was not dependent on FOXO1 (Fig. 6). In fact, in lymphocytes, glucocorticoid-induced cell death was reversed by knockdown of BCL2-like 11 (apoptosis facilitator) (BIM) [31].

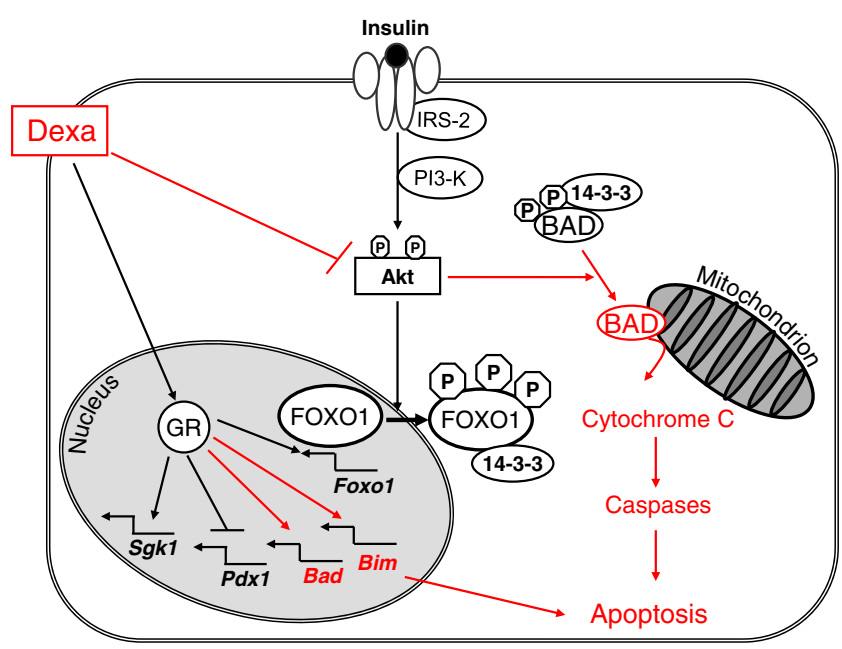

Fig. 6 Dexamethasone-induced beta cell death is not initiated by activation of FOXO1. Dexamethasone increases $\mathrm{Sgk1}$, Foxol, Bim and $\mathrm{Bad}$ expression through glucocorticoid-receptor activation and concomitantly reduces $P d x 1$ mRNA. The reduced phosphorylation of AKT under excess of glucocorticoids does not activate FOXO1-dependent apoptosis but reduces BAD phosphorylation [11], indicating that dexamethasone promotes beta cell death directly through changes of mitochondrial BCL-2 proteins. Dexa, dexamethasone; GR, glucocorticoid receptor; PI3K, phosphatidylinositide 3-kinase 
Our previous study revealed dexamethasone-dependent dephosphorylation of the pro-apoptotic BCL-2 protein BAD, which is a substrate of AKT [11]. On dephosphorylation BAD translocates to mitochondria, accomplishing an essential step of mitochondrial-linked apoptotic cell death [32]. In view of reduced AKT phosphorylation in dexamethasone-treated INS-1E cells, BAD seems to be more sensitive towards inhibition of AKT than FOXO1 (Fig. 6). An $80 \%$ reduction in a single or two $A k t$ isoforms by siRNA did not impair FOXO1 phosphorylation. Quantification of relative Akt mRNA levels indicates that $20 \%$ of total AKT activity is sufficient to maintain FOXO1 in a phosphorylated state. The concomitant knockdown of all three AKT isoforms, AKT1, AKT2 and AKT3, finally suggests that each AKT isoform is able to compensate for the loss of AKT activity. These results were confirmed by the pan AKT inhibitor Akti-1/2, which effectively dephosphorylated FOXO1, leading to its nuclear accumulation. The addition of Akti-1/2 for $2 \mathrm{~h}$ to cells which were pre-exposed to dexamethasone for $22 \mathrm{~h}$ potentiated beta cell death, indicating additive effects through distinct pathways. A synergistic action of glucocorticoid receptor and FOXO1 has been described in muscle cells, pointing towards an independent activation of these pathways [33]. Here, we found that interaction of glucocorticoid receptor and FOXO1 leads to a potentiation of Bim expression, promoting mitochondrialdependent apoptosis. During the development of diabetes such a synergistic effect could be induced by NEFA as they stimulate nuclear translocation of FOXO1 [34, 35].

The second surprising finding is that SGK1 does not contribute to phosphorylation of FOXO1 under glucocorticoid excess. This is contradictory to previous studies, in which SGK1 was found to phosphorylate FOXO1 at Ser256 and Ser319 [36, 37]. The expression of $S g k 1$ is highly regulated in insulin-secreting cells and glucocorticoids augment mRNA and protein of SGK1 up to 20-50fold [18]. However, downregulation of SGK1 by siRNA along with dexamethasone treatment did not reduce the phosphorylation of FOXO1. Moreover, overexpression of a constitutively active or inactive SGK1 (SGK1SD and SGK1KN) did not affect FOXO1 phosphorylation. The commercially available inhibitor was also ineffective. These observations underline that AKT isoforms are the main regulators of FOXO1 in insulin-secreting cells.

The dissociation between cell death and FOXO1 activation in insulin-secreting cells became obvious when phosphorylation and subcellular distribution of the transcription factor were simultaneously analysed. Our study unfolds an important molecular mechanism which determines subcellular localisation of FOXO1. Nuclear activity of FOXO1 depends on additional modifications such as acetylation [38]. Further studies are needed to understand the regulation of nuclear FOXO1 activity. Concerning the role of FOXO1 in beta cells, a significant study has recently been performed with beta cell-specific deletion of FOXO1 in mice [39]. This study, published by Talchai and coworkers, shows that FOXO1 regulates differentiation of pancreatic endocrine cells rather than apoptosis. In addition, our results demonstrate the unique and promiscuous role of AKT isoforms for FOXO1 phosphorylation and reveal that AKT activity is decisive for beta cell survival.

Glucocorticoids regulate physiological blood glucose concentration by mobilising glucose from hepatocytes [40]. An excess of glucocorticoids, however, as during immunosuppressive drug treatment, leads frequently to the development of hyperglycaemia and steroid diabetes [1]. Apart from the induction of insulin resistance, glucocorticoids compromise beta cell function and induce beta cell death. The present study sheds light on the fact that glucocorticoids prime the cells for the cytotoxic effects seen on impairment of AKT signalling while denying the involvement of FOXO1 activation in glucocorticoid-induced beta cell apoptosis.

Acknowledgements We thank S. Haug (Institute for Diabetes Research and Metabolic Diseases of the Helmholtz Centre Munich at the University of Tübingen, Partner of the German Centre for Diabetes Research, Tübingen, Germany) and A. Janessa (University Hospital of Tübingen, Department of Internal Medicine, Tübingen, Germany) for skilled technical help.

Funding The study was supported by DFG grants UL140/7-2 and GRK1302/1 and by a grant from the German Federal Ministry of Education and Research (BMBF) to the German Centre for Diabetes Research. G. Kaiser is a fellow of the international graduate school GRK1302/1 and this study is part of her PhD thesis.

Duality of interest The authors declare that there is no duality of interest associated with this manuscript.

Contribution statement GK and FG performed experiments, analysed data, wrote and revised the manuscript. DM, SB and BF performed experiments and analysed data and revised the manuscript; NS-S generated the SGK1 plasmids and designed experiments and revised the manuscript. FL and $\mathrm{H}-\mathrm{UH}$ contributed to the design of experiments, interpretation of results and revised the manuscript. SU designed the study, analysed data and drafted, revised and edited the manuscript. All authors approved the final version of the manuscript.

\section{References}

1. Schäcke H, Docke WD, Asadullah K (2002) Mechanisms involved in the side effects of glucocorticoids. Pharmacol Ther 96:23-43

2. Tounian P, Schneiter P, Henry S, Delarue J, Tappy L (1997) Effects of dexamethasone on hepatic glucose production and fructose metabolism in healthy humans. Am J Physiol 273:E315-E320

3. Andrews RC, Walker BR (1999) Glucocorticoids and insulin resistance: old hormones, new targets. Clin Sci (Lond) 96:513-523

4. Beaudry JL, Riddell MC (2012) Effects of glucocorticoids and exercise on pancreatic beta-cell function and diabetes development. Diabetes Metabol Res Rev 28:560-573

5. Talchai C, Lin HV, Kitamura T, Accili D (2009) Genetic and biochemical pathways of beta-cell failure in type 2 diabetes. Diabetes Obes Metabol 11(Suppl 4):38-45 
6. Kobayashi M, Kikuchi O, Sasaki T et al (2012) FoxO1 as a doubleedged sword in the pancreas: analysis of pancreas- and beta-cellspecific FoxO1 knockout mice. Am J Physiol Endocrinol Metab 302:E603-E613

7. Kitamura T, Nakae J, Kitamura Y et al (2002) The forkhead transcription factor Foxo1 links insulin signaling to $\mathrm{Pdx} 1$ regulation of pancreatic beta cell growth. J Clin Invest 110:1839-1847

8. Kitamura YI, Kitamura T, Kruse JP et al (2005) FoxO1 protects against pancreatic beta cell failure through NeuroD and MafA induction. Cell Metab 2:153-163

9. Zhang X, Yong W, Lv J et al (2009) Inhibition of forkhead box O1 protects pancreatic beta-cells against dexamethasone-induced dysfunction. Endocrinol 150:4065-4073

10. Wrede CE, Dickson LM, Lingohr MK, Briaud I, Rhodes CJ (2002) Protein kinase B/Akt prevents fatty acid-induced apoptosis in pancreatic beta-cells (INS-1). J Biol Chem 277:49676-49684

11. Ranta F, Avram D, Berchtold S, Düfer M, Drews G, Lang F, Ullrich S (2006) Dexamethasone induces cell death in insulin secreting cells, an effect reversed by Exendin-4. Diabetes 55:1380-1390

12. Lambillotte C, Gilon P, Henquin JC (1997) Direct glucocorticoid inhibition of insulin secretion. An in vitro study of dexamethasone effects in mouse islets. J Clin Invest 99:414-423

13. Philippe J, Missotten M (1990) Dexamethasone inhibits insulin biosynthesis by destabilizing insulin messenger ribonucleic acid in hamster insulinoma cells. Endocrinol 127:1640-1645

14. Gremlich S, Roduit R, Thorens B (1997) Dexamethasone induces posttranslational degradation of GLUT2 and inhibition of insulin secretion in isolated pancreatic beta cells. Comparison with the effects of fatty acids. J Biol Chem 272:3216-3222

15. Delaunay F, Khan A, Cintra A et al (1997) Pancreatic beta cells are important targets for the diabetogenic effects of glucocorticoids. J Clin Invest 100:2094-2098

16. Avram D, Ranta F, Hennige AM et al (2008) IGF-1 protects against dexamethasone-induced cell death in insulin secreting INS-1 cells independent of Akt/PKB phosphorylation. Cell Physiol Biochem 21:455-462

17. Hamamdzic D, Duzic E, Sherlock JD, Lanier SM (1995) Regulation of alpha 2-adrenergic receptor expression and signaling in pancreatic beta-cells. Am J Physiol 269:E162-E171

18. Ullrich S, Berchtold S, Ranta F et al (2005) Serum- and glucocorticoid-inducible kinase 1 (SGK1) mediates glucocorticoidinduced inhibition of insulin secretion. Diabetes 54:1090-1099

19. Henke G, Maier G, Wallisch S, Boehmer C, Lang F (2004) Regulation of the voltage gated $\mathrm{K}+$ channel Kv1.3 by the ubiquitin ligase Nedd4-2 and the serum and glucocorticoid inducible kinase SGK1. J Cell Physiol 199:194-199

20. Friedrich B, Weyrich P, Stancakova A et al (2008) Variance of the SGK1 gene is associated with insulin secretion in different European populations: results from the TUEF, EUGENE2, and METSIM studies. PLoS One 3:e3506

21. Van der Heide LP, Hoekman MF, Smidt MP (2004) The ins and outs of FoxO shuttling: mechanisms of FoxO translocation and transcriptional regulation. Biochem J 380:297-309

22. Burgering BM, Medema RH (2003) Decisions on life and death: FOXO Forkhead transcription factors are in command when PKB/ Akt is off duty. J Leukoc Biol 73:689-701

23. Rena G, Woods YL, Prescott AR et al (2002) Two novel phosphorylation sites on FKHR that are critical for its nuclear exclusion. EMBO J 21:2263-2271
24. Alikhani M, Alikhani Z, Graves DT (2005) FOXO1 functions as a master switch that regulates gene expression necessary for tumor necrosis factor-induced fibroblast apoptosis. J Biol Chem 280:12096-12102

25. Lindsley CW, Zhao Z, Leister WH et al (2005) Allosteric Akt (PKB) inhibitors: discovery and SAR of isozyme selective inhibitors. Bioorg Med Chem Lett 15:761-764

26. Lazebnik YA, Kaufmann SH, Desnoyers S, Poirier GG, Earnshaw WC (1994) Cleavage of poly(ADP-ribose) polymerase by a proteinase with properties like ICE. Nature 371:346-347

27. Arumugam R, Horowitz E, Lu D et al (2008) The interplay of prolactin and the glucocorticoids in the regulation of beta-cell gene expression, fatty acid oxidation, and glucose-stimulated insulin secretion: implications for carbohydrate metabolism in pregnancy. Endocrinol 149:5401-5414

28. Poulsen RC, Carr AJ, Hulley PA (2011) Protection against glucocorticoid-induced damage in human tenocytes by modulation of ERK, Akt, and forkhead signaling. Endocrinol 152:503-514

29. Miao H, Zhang Y, Lu Z, Liu Q, Gan L (2012) FOXO1 involvement in insulin resistance-related pro-inflammatory cytokine production in hepatocytes. Inflamm Res 61:349-358

30. Puthanveetil P, Wang Y, Wang F, Kim MS, Abrahani A, Rodrigues B (2010) The increase in cardiac pyruvate dehydrogenase kinase-4 after short-term dexamethasone is controlled by an Akt-p38-forkhead box other factor-1 signaling axis. Endocrinol 151:2306-2318

31. Abrams MT, Robertson NM, Yoon K, Wickstrom E (2004) Inhibition of glucocorticoid-induced apoptosis by targeting the major splice variants of BIM mRNA with small interfering RNA and short hairpin RNA. J Biol Chem 279:55809-55817

32. Datta SR, Dudek H, Tao X et al (1997) Akt phosphorylation of BAD couples survival signals to the cell-intrinsic death machinery. Cell 91:231-241

33. Waddell DS, Baehr LM, van den Brandt J et al (2008) The glucocorticoid receptor and FOXO1 synergistically activate the skeletal muscle atrophy-associated MuRF1 gene. Am J Physiol Endocrinol Metab 295:E785-E797

34. Hennige AM, Ranta F, Heinzelmann I et al (2010) Overexpression of kinase-negative protein kinase Cdelta in pancreatic beta-cells protects mice from diet-induced glucose intolerance and beta-cell dysfunction. Diabetes 59:119-127

35. Martinez SC, Tanabe K, Cras-Meneur C, Abumrad NA, BernalMizrachi E, Permutt MA (2008) Inhibition of Foxo1 protects pancreatic islet beta-cells against fatty acid and endoplasmic reticulum stress-induced apoptosis. Diabetes 57:846-859

36. Feroze-Zaidi F, Fusi L, Takano M et al (2007) Role and regulation of the serum- and glucocorticoid-regulated kinase 1 in fertile and infertile human endometrium. Endocrinol 148:5020-5029

37. Di Pietro N, Panel V, Hayes S et al (2010) Serum- and glucocorticoid-inducible kinase 1 (SGK1) regulates adipocyte differentiation via forkhead box O1. Mol Endocrinol 24:370-380

38. Chae HD, Broxmeyer HE (2011) SIRT1 deficiency downregulates PTEN/JNK/FOXO1 pathway to block reactive oxygen speciesinduced apoptosis in mouse embryonic stem cells. Stem Cell Dev 20:1277-1285

39. Talchai C, Xuan S, Lin HV, Sussel L, Accili D (2012) Pancreatic beta cell dedifferentiation as a mechanism of diabetic beta cell failure. Cell 150:1223-1234

40. Kraus-Friedmann N (1984) Hormonal regulation of hepatic gluconeogenesis. Physiol Rev 64:170-259 